\title{
Toints
}

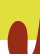

\section{Treatment of pincer-type femoroacetabular impingement}

\author{
ETTORE SABETTA, EDOARDO SCARAVELLA
}

Arcispedale Santa Maria Nuova, Reggio Emilia, Italy

\begin{abstract}
Pincer femoroacetabular impingement (FAI) consists of pathological contact between the acetabular labrum and rim and the femoral head-neck junction. Manifold conditions underlie pincer FAI: anatomical abnormalities, malorientation of the acetabulum, torsional abnormalities of the neck and femoral shaft (these defects can be constitutional, post-traumatic or post-surgical), and involvement in sports characterized by repeated and sudden maximum joint excursions. In a high percentage of cases, pincer FAI is associated with cam FAI.

The aims of surgical treatment of pincer FAI are to eliminate the cause of the contact and repair the joint damage; the surgery may be open or arthroscopic, performed with an articular or extra-articular approach. Recently, arthroscopic treatment of FAI had a rapid and widespread diffusion due to the advantages it offers compared with the open technique. Arthroscopic treatment can repair the joint damage and in some cases, characterized by minor deformity, compensate for extra-articular defects. The acetabular labrum must always be preserved and sutured; only in extreme cases can it be sacrificed. Post-operative mobilization must respect the healing time of the labral repair.
\end{abstract}

Key Words: pincer, femoroacetabular impingement, hip arthroscopy, osteochondroplasty, acetabular labrum.

\footnotetext{
Corresponding Author:

Edoardo Scaravella, MD

Arcispedale Santa Maria Nuova

Viale Risorgimento, 80

42123 Reggio Emilia, Italy

E-mail: scaramem@gmail.com
}

\section{Introduction}

Femoroacetabular impingement (FAI) consists of pathological contact of the acetabular labrum and rim against the femoral head-neck junction during physiological movements of the hip $(1,2)$.

The acetabular labrum is a ring of fibrocartilage, comparable to the menisci of the knee, being elastic and having a good capacity for absorbing shocks and mechanical stresses. It is thus able to withstand for a considerable time the stresses caused by the pathological contact. The fact that the labrum is richly innervated by nociceptors justifies the onset of pain before the appearance of tears or degenerative changes.

\section{The pathomechanics of pincer FAI}

Pincer FAI involves repeated and frequent contact between the acetabular labrum and the femoral neck. The conditions that underlie pincer FAI are manifold. It can be caused by anatomical abnormalities of the acetabulum or the femoral neck, by malorientation of the acetabulum, and by torsional abnormalities of the neck and femoral shaft. These orientation and torsion abnormalities can be constitutional or post-traumatic, e.g. the result of femoral or acetabular fractures. They may also be secondary to surgical procedures, in particular to acetabular and femoral osteotomies. Finally, in subjects who practice certain sports, such as gymnastics, martial arts and dance, in which the athletic movements are characterized by repeated and sudden maximum joint excursions, pincer FAI can have a functional cause. In a high percentage of cases pincer FAI is associated with cam FAI.

The most serious damage caused by pincer FAI occurs at the level of the acetabular cartilage at its junction with the labrum (Fig. 1). The cartilage lesion is localized in the area where the contact occurs, which is 


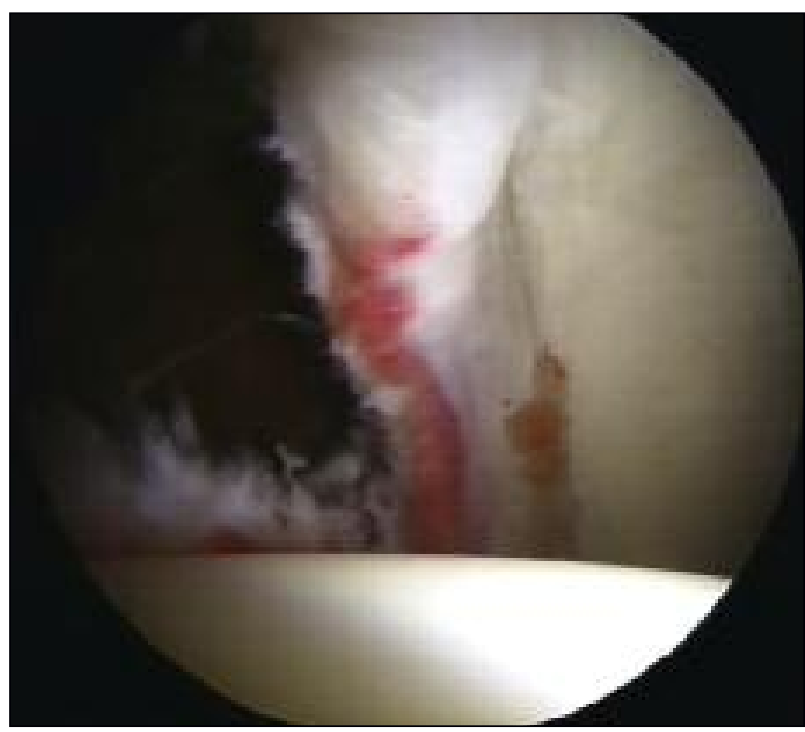

Fig. 1. Labral contusion in pincer FAI.

usually the anterosuperior portion of the acetabulum. In the early stages the cartilage remains in continuity with the labrum, but is separated from the bone surface. Typical of this phase is the wave sign, visible in arthroscopy. On compressing the labrum with a blunt instrument, the cartilage is lifted from the underlying bone surface in a wave-like manner. If the causes of the contact are not removed, the cartilage delamination extends progressively in a cranio-caudal direction toward the center of the joint; this results in separation of the cartilage from the labrum and the formation of unstable flaps. It is generally only in the advanced stage that the labrum becomes separated from the edge of the acetabulum, and there is serious labral degeneration and more or less extensive tearing.

\section{Diagnosis}

The clinical diagnosis of pincer FAI is based on the patient-reported medical history and on specific clinical tests. In typical cases of anterior pincer FAI, the pain is elicited by placing the hip in flexion-adduction and internal rotation. In posterior pincer FAI, the opposite direction of force, i.e. in hyperextensionexternal rotation, evokes posterior pain.

Imaging of pincer FAI is based on standard radiography examinations, using the anteroposterior (AP) view of the pelvis, and the axial view or frog-legs view of the hip. Computed tomography or magnetic resonance arthrography can be performed to evaluate the articular cartilage and the acetabular labrum.

\section{Treatment}

The aims of surgical treatment of pincer FAI are twofold: to eliminate the cause of the contact and to repair the joint damage it has caused.

Although arthroscopic treatment of FAI recently had a rapid and widespread diffusion due to its advantages compared with the open technique, its use should not be overemphasized. Arthroscopic treatment can repair the joint damage and in some cases (characterized by minor deformity), compensate for extra-articular defects; but when the pincer FAI is caused by acetabular malorientation or major femoral torsional abnormalities, surgery must be extra-articular, performed with the open technique. In the first case, periacetabular osteotomy, performed with the Bernese technique $(3,4)$, may be indicated. In presence of femoral defects (femoral neck varus/valgus, anteversion/retroversion, diaphyseal torsion vice), adequate osteotomy correction is indicated. In some cases, both procedures, intraand extra-articular, are necessary. In such situations, the open technique can be chosen to treat both pathologies, or arthroscopy (used to address the intra-articular pathology) can be associated with the open technique for extra-articular correction.

As already indicated, the treatment of FAI pincer is divided into two phases: 1) removal of the causes; 2) repair of the lesions. If the position of the femoral epiphysis in relation to the joint surface is higher than normal, resulting in acetabular over-coverage, we proceed with osteochondroplasty. This procedure involves smoothing, after detachment of the labrum, the acetabular rim in the tract where there is contact, using a sharp chisel in the open technique or a circular cutter in the arthroscopic procedure.

Acetabular osteochondroplasty eliminates the contact with the femoral neck, but, on the other hand, reduces the loading surface of the acetabulum against the femoral epiphysis. Therefore, it should be practiced only in cases of partial acetabular over-coverage, generally anterior-superior or global (coxa profunda, pro- 
trusio acetabuli). If practiced in a normal acetabulum or a hypoplastic or even dysplastic one, osteochondroplasty causes reduction of the loading area resulting in possible secondary overload degeneration. It can also cause micro-instability or severe instability with a risk of dislocation in dysplastic hips (5).

The extent of acetabular osteochondroplasty has to be carefully determined preoperatively and during surgery. Preoperative planning should be based on the AP radiograph of the pelvis and on the Lequesne false profile (an oblique view) $(6,7)$. The AP view of the pelvis has to be correct without defects of rotation or inclination, such that the apex of the coccyx is at a distance of $1-3 \mathrm{~cm}$ from symphysis pubis. On this image we evaluate the center edge (CE) angle of Wiberg, whose normal values are between $25^{\circ}$ and $40-45^{\circ}$. With a good approximation, a $1 \mathrm{~mm}$ lowering of the acetabular edge coincides with a $1^{\circ}$ reduction of Wiberg's CE angle. A similar assessment should be performed in the Lequesne projection. In special cases, when standard radiology is not exhaustive, a CT scan can be performed, ideally with 3D reconstruction.

Intraoperative assessment of the extent and depth of acetabular osteochondroplasty is more complex, as there are no safe reference parameters. The experience gained with successive cases allows the surgeon to achieve a good level of reliability in visual judgments. The intraoperative landmarks used for locating where to intervene, and to what extent and depth, can vary. First, it is necessary to find the line of cartilage fusion with the three bones of the pelvis: ilium, ischium and pubis. This line gives an indication for the " 12 o'clock" position. Arthroscopic visual exploration (Fig. 2) of the labrum and its junction with the acetabular cartilage can be used to reliably determine the extent of the contact zone. The circumference of the acetabulum is likened to a clock dial and the extent of the lesion is indicated by using the time difference between its start and end point (f.e., in a right hip, the lesion can extend from 12 to 15 hours if the contact is in the anterosuperior region). A chondral lesion, if present, should be described accurately indicating its topography, extension and depth.

Acetabular osteochondroplasty requires exposure of the acetabular bony edge in the area that should be removed by use of motorized shaver and radiofrequencies. Once the bone has been exposed, a $5.5 \mathrm{~mm}$ circular cutter is used to blunt the acetabular edge,

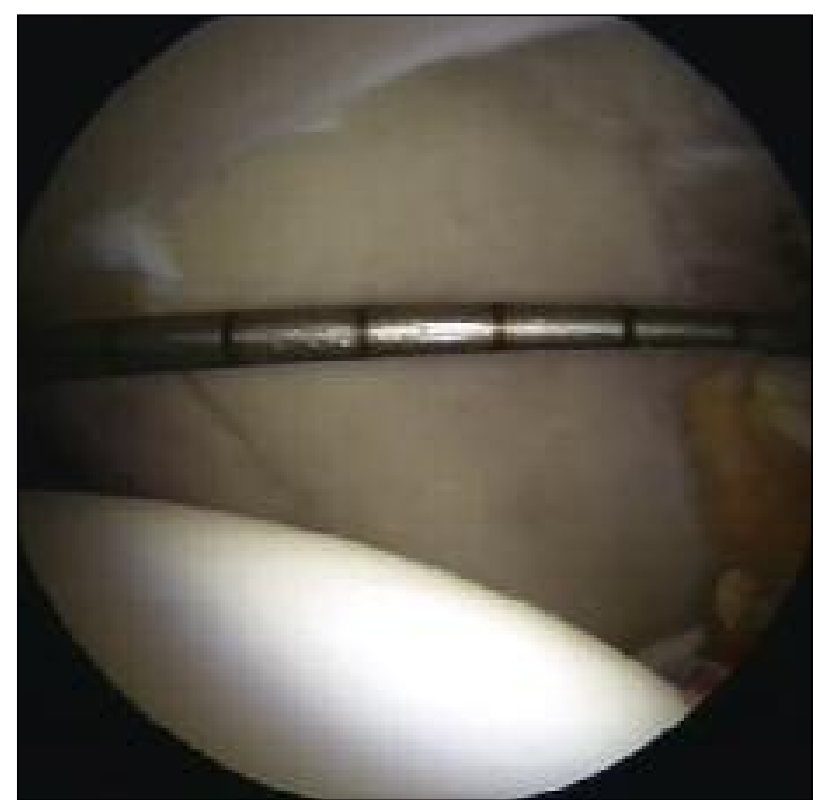

Fig. 2. Twelve o'clock measurement of acetabular coverage.

after releasing the labrum. As a first step, the bone edge should be notched at different points sinking the circular cutter into the bone, just enough to mark the millimeters that it is intended to remove. Knowing the diameter of the sphere allows the surgeon to reach the desired depth with good reliability. The next step involves removing, with the circular cutter, the bone between successive notches, in order to obtain a rectilinear, uniform and less prominent plane.

If the depth of the acetabular osteochondroplasty is $2-3 \mathrm{~mm}$, it is not necessary to detach the labrum, but this is progressively undermined up to the acetabular cartilage and then stabilized with suture anchors. For more substantial acetabular osteochondroplasties, it is necessary to detach the labrum from the bone and cartilage, re-inserting it at the end, after the other procedures.

A useful benchmark measurement for checking the depth of the osteocondroplasty is the distance between the apex of the acetabular fossa pointed at 12 o'clock and the edge of the acetabulum. To obtain this measurement it is sufficient use a hook stylus graduated in millimeters. The measurement should be recorded before starting osteochondroplasty, and then monitored during the procedure. The distance between the tip of the ogive of the acetabular fossa and 
the edge of the acetabulum should not be less than 25 $\mathrm{mm}$. As described above, 1 degree in Wiberg's CE angle corresponds to $1 \mathrm{~mm}$, therefore if the distance is not allowed to fall below $25 \mathrm{~mm}$ with acetabular osteochondroplasty, one can be certain that the acetabular coverage has not been excessively reduced.

The osteochondroplasty is followed by stabilization of the labrum, using small diameter (from $2.3 \mathrm{~mm}$ to 2.9 $\mathrm{mm}$ ) biodegradable suture anchors single loaded with high-strength permanent sutures. The anchors should be spaced about 1 centimeter apart. The most difficult step is inserting them into the subchondral bone, close to the acetabular cartilage, so that the labrum adheres firmly to the bone, but not in an elevated position with respect to the edge of the acetabulum, so as not to lose the "seal" effect. If the angle of the drill used for the creation of the anchor hole and subsequently the angle of the bone anchor inserter are not correct, there is the risk that the anchor will emerge on the articular surface. For this reason, both during drilling and after insertion of the anchor, it is necessary to check the articular surface of the acetabulum. When knotting the sutures of the labrum, care must be taken not to tighten them excessively, to prevent the labrum from rising above the articular surface, otherwise its sealing function will be lost. After suturing and before releasing the limb traction, the stability of the labrum must be checked with a hooked probe. Some surgeons prefer to release the traction before the suturing of the labrum, tightening the sutures with the labrum perfectly in contact with femoral epiphysis, so that the seal effect of the labrum is restored.

The acetabular labrum must always be preserved and sutured; it is sacrificed only in cases of extreme degeneration or if it is extensively ossified, as is sometimes observed, especially, in coxa profunda. Several studies have shown that the prognosis is better when the labrum is present (8-11). If pincer FAI manifests itself in the presence of a hypoplastic acetabulum and/or overload, acetabular osteochondroplasty should not be performed, because it further will reduce the surface. In such cases, osteochondroplasty must be performed on the femur, at the head/neck junction, in order to increase the offset in this area. During surgery, dynamic tests of flexion-adduction-internal rotation must be performed, to check the correction of the contact.

Post-operative mobilization must respect the healing time of the labral repair. For the first 2-3 weeks, external rotation with limb extended is precluded. Internal rotation is allowed, but to only a few degrees and flexion to $90^{\circ}$. From the $3^{\text {rd }}-4^{\text {th }}$ week, the range of motion is progressively increased in all planes until the complete joint recovery. Loading is not contraindicated in the healing phase of the labrum. For the first two weeks crutches are used to protect against excessive loading. The return to contact sports is allowed from $3^{\text {rd }}-4^{\text {th }}$ month in absence of other joint injuries.

\section{References}

1. Leunig M, Beaulé PE, Ganz R. The concept of femoroacetabular impingement: current status and future perspectives. Clin Orthop Relat Res. 2009;467:616-622.

2. Leunig M, Ganz R. FAI - concept and etiology. Orthopade. 2009;38:394-401.

3. Leunig M, Ganz R. Evolution of technique and indications for the Bernese periacetabular osteotomy. Bull NYU Hosp Jt Dis. 2011;69 Suppl 1:S42-46.

4. Ganz R, Leunig M. Osteotomy and the dysplastic hip: the Bernese experience. Orthopedics. 2002;25:945-946.

5. Ross JR, Clohisy JC, Baca G, Sink E; ANCHOR Investigators. Patient and disease characteristics associated with hip arthroscopy failure in acetabular dysplasia. J Arthroplasty. 2014;29 (9 Suppl):160-163.

6. Lequesne M, Bellaïche L. Anterior femoroacetabular impingement: an update. Joint Bone Spine. 2012;79:249-255.

7. Bellaïche L, Lequesne M, Gedouin JE, Laude F, Boyer T; French Arthroscopy Society. Imaging data in a prospective series of adult hip pain in under-50 year-olds. Orthop Traumatol Surg Res. 2010;96:S44-52.

8. Byrd JW, Jones KS. Primary repair of the acetabular labrum: outcomes with 2 years' follow-up. Arthroscopy. 2014;30:588592.

9. Leunig M, Ganz R. The evolution and concepts of joint-preserving surgery of the hip. Bone Joint J. 2014;96-B:5-18.

10. Jackson TJ, Watson J, La Reau JM, Domb BG. Periacetabular osteotomy and arthroscopic labral repair after failed hip arthroscopy due to iatrogenic aggravation of hip dysplasia. Knee Surg Sports Traumatol Arthrosc. 2014;22:911-914.

11. Geyer MR, Philippon MJ, Fagrelius TS, Briggs KK. Acetabular labral reconstruction with an iliotibial band autograft: outcome and survivorship analysis at minimum 3-year follow-up. Am J Sports Med. 2013;41:1750-1756. 